\title{
Hoarseness, cough and cervical lymphadenopathy
}

\author{
A.J. Turk*, T. Brack*, C. Alfaré*, A. Gaspert" ${ }^{\#}$ and E.W. Russi*
}

\section{CASE HISTORY}

A 43-yr-old native Brazilian female complained of a sore throat, recent onset of hoarseness, progressive pain and bilateral swelling of the neck during the past 6 months, when she presented at the University Hospital of Zurich (Zurich, Switzerland) for the first time. The patient had moved to Switzerland 5 yrs before, had been a heavy smoker for the past 20 yrs and coughed chronically. She reported a short episode of minor haemoptysis $2 \mathrm{yrs}$ previously that had ceased spontaneously. The patient suffered no fever or night sweats, but had lost $2 \mathrm{~kg}$ of weight during the past 2 months, despite a normal appetite. Five months previously, she had spent 3 months in a town south of the capital Brasilia, where she was treated with sulphamethoxazole and trimethoprim for

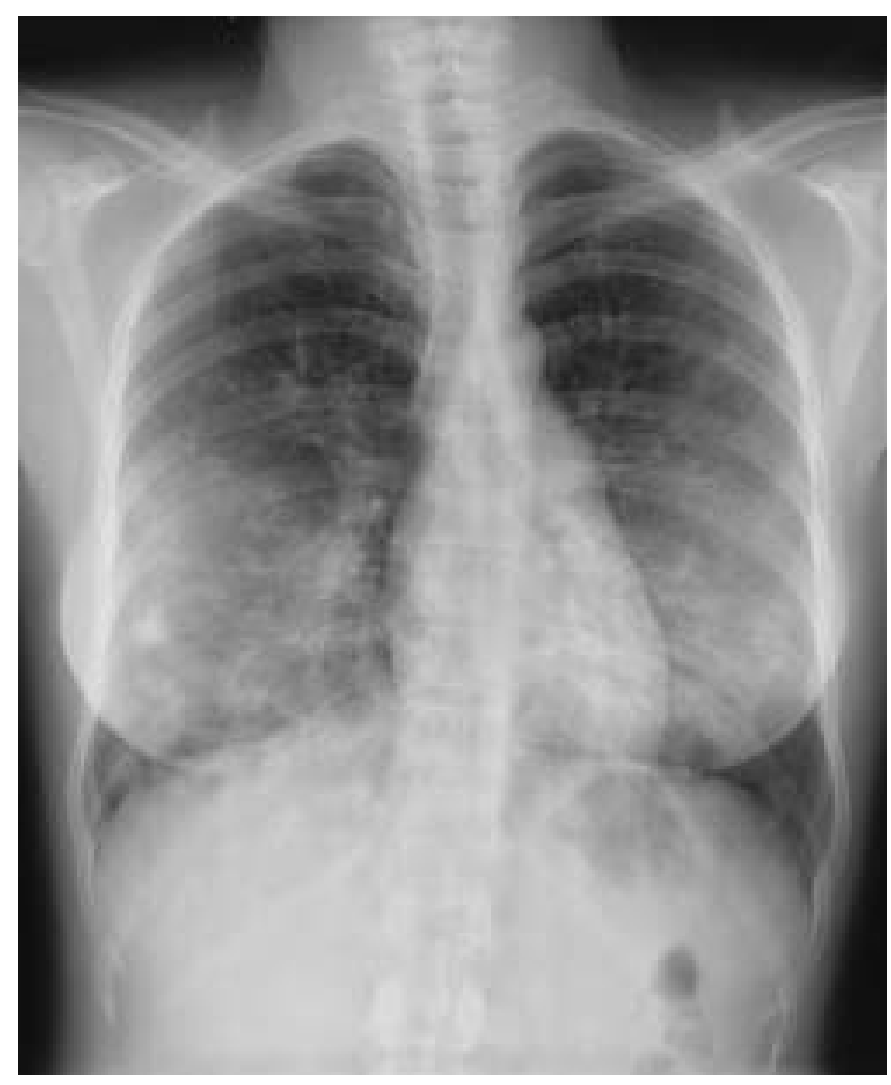

FIGURE 1. Chest radiograph taken 3 months prior to the current presentation. pneumonia. After a transient recovery, the cough, sore throat and cervical swelling reappeared.

The patient's past medical history was unremarkable for severe diseases or surgery, other than a bilateral silicon breast implant. A year before, the patient stopped taking four different drugs to lose weight, containing substances such as benzodiazepines, fluoxetine, metoclopramide and diethylpropione, a stimulant that is commonly used as an appetite suppressant in Brazil.

The clinical examination was remarkable for enlarged, painful cervical lymph nodes and a few bibasilar rales. The patient had bilateral silicon breast implants. Her temperature was normal, white blood cell count was $10.09 \times 10^{-3} \cdot \mu \mathrm{L}^{-1}$ (without left shift), C-reactive protein was $42 \mathrm{mg} \cdot \mathrm{L}^{-1}$ (normal: $<5 \mathrm{mg} \cdot \mathrm{L}^{-1}$ ) and HIV antibodies were negative. The chest radiograph (fig. 1) taken 3 months previously in Brazil showed dense bilateral alveolar infiltrates, predominantly in the mid-zone of the lungs. A recent computed tomography (CT) scan is shown in figure 2 . Fine-needle aspiration of a cervical lymph node demonstrated necrotising epitheloid granulomas without acid-fast bacilli, and the culture of the aspirate did not grow mycobacteria. Bronchoscopy (fig. 3) with bronchoalveolar lavage (BAL) and transbronchial biopsies were performed (figs 4 and 5).

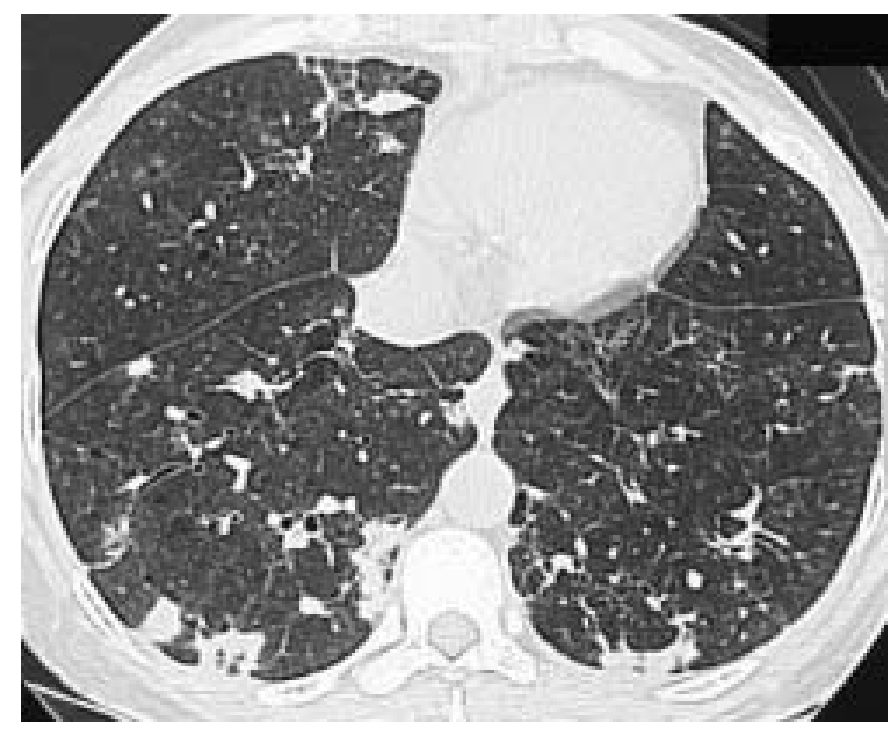

FIGURE 2. Thin-section computed tomography at the level of the lower lobes.

*Pulmonary Division, Dept of Internal Medicine, and \#Dept of Pathology, University Hospital of Zurich, Zurich, Switzerland.

CORRESPONDENCE: A.J. Turk, Pulmonary Division, Dept of Internal Medicine, University Hospital of Zurich, Rämistrasse 100, CH-8091 Zürich, Switzerland. Fax: 41 12554451. E-mail: alexander.turk@usz.ch 


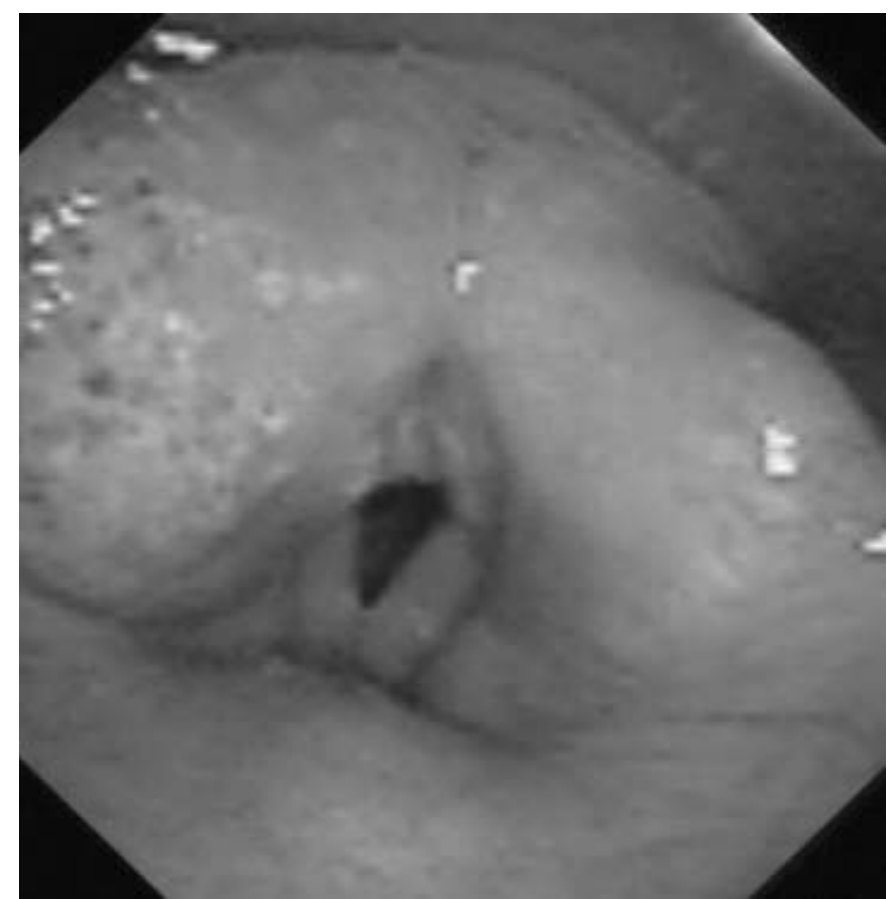

FIGURE 3. View of the larynx and vocal cords during bronchoscopy.

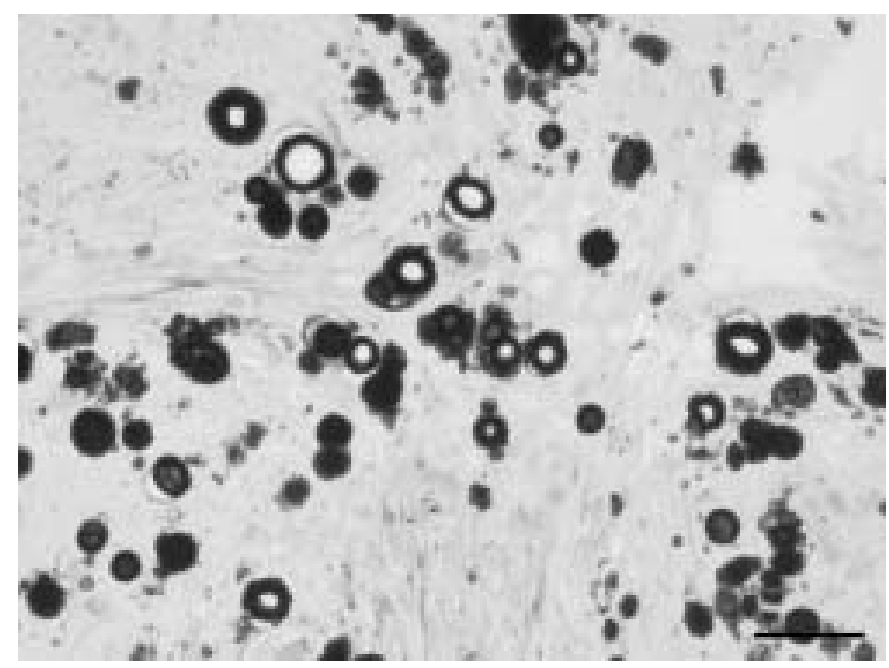

FIGURE 5. Transbronchial lung biopsy (Grocott's hexamine-silver stain). Scale bar $=20 \mu \mathrm{m}$.
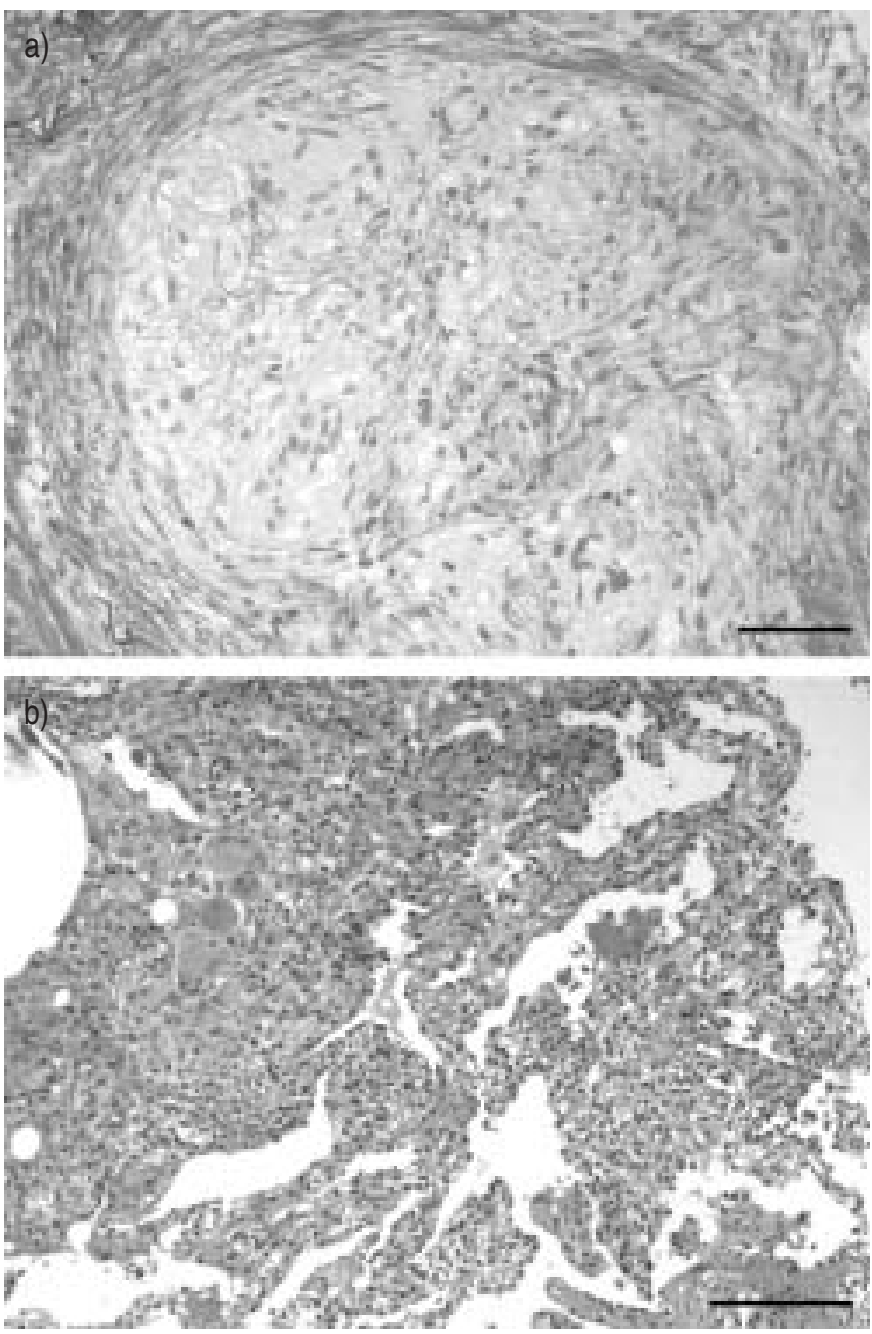

FIGURE 4. Transbronchial lung biopsy with a) Elastica van Gieson stain, and b) haematoxylin and eosin stain. Scale bars $=50 \mu \mathrm{m}$ (a) and $200 \mu \mathrm{m}$ (b)

BEFORE TURNING THE PAGE, INTERPRET THE COMPUTED TOMOGRAPHY SCAN, THE BRONCHOSCOPIC VIEW OF THE LARYNX AND THE LUNG BIOPSY FINDINGS, AND SUGGEST A DIAGNOSIS. 


\section{INTERPRETATION}

\section{Chest radiograph}

The chest radiograph shows dense bilateral alveolar infiltrates, predominantly in the mid-zone of the lungs (fig. 1).

\section{Computed tomography}

The CT scan reveals small, peripheral and spiculated consolidations with signs of architectural distortion. Some consolidations are surrounded by confluating air-space nodules and subtle areas of ground-glass opacities (fig. 2).

\section{Bronchoscopy}

Bronchoscopy revealed an inflamed, enlarged, oedematous larynx with a cobblestone-like mucosa (fig. 3) and a purulent bronchitis. BAL was remarkable for intracellular fungal spores in macrophages.

\section{Pathology}

The transbronchial biopsies demonstrated a granulomatous and interstitial inflammation with macrophages and multinucleated giant cells containing fungal spores (figs 4 and 5).

\section{Culture of BAL}

The fungal cultures grew Paracoccidioides brasiliensis.

\section{Diagnosis: Paracoccidiomycosis with involvement of the cervical lymph nodes, larynx and lung.}

\section{Clinical course}

The patient was treated with $2 \times 100 \mathrm{mg} \cdot \mathrm{day}^{-1}$ of itraconazole over 6 months, and promptly recovered from the cough and the hoarseness. The cervical lymphadenopathy also disappeared. A CT scan at follow-up was unremarkable except for some interstitial scarring. Retrospectively, fungal spores could be identified in the Grocott's hexamine-silver stain of the fineneedle aspirate of the cervical lymph node.

\section{DISCUSSION}

Paracoccidiomycosis, also known as South American blastomycosis, is considered a primary systemic mycosis in contrast to the opportunistic mycoses that only affect immunocompromised patients. Other primary systemic mycoses are histoplasmosis, coccidiomycosis and North American blastomycosis. Their common habitat is the soil. The spores are inhaled and cause a primary pulmonary mycosis [1]. Primary systemic mycoses are also called endemic mycoses because they occur only in certain geographical regions. Paracoccidiomycosis occurs exclusively in South America, where South Mexico $\left(23^{\circ} \mathrm{N}\right.$ latitude) and Argentina ( $34.5^{\circ} \mathrm{S}$ latitude) build the northern and southern border of the endemic area, respectively. Approximately $60 \%$ of the cases occur in Brazil, while fewer cases are seen in Colombia, Venezuela, Argentina and Peru. Areas with the highest incidences are subtropical rainforests and damp mountain forests. The disease does not occur in hot, dry areas or in rainforests around the equator where the temperature rises above $25^{\circ} \mathrm{C}$.

Paracoccidiomycosis is not transmitted among humans. Most patients with paracoccidiomycosis are male agricultural workers. The outbreak of the disease is favoured by malnutrition and alcoholism. Tuberculosis (TB) occurs as a co-infection in $12 \%$ of the cases [2]. After inhalation of the fungal spores, there is often an asymptomatic or subclinical primary pulmonary paracoccidiomycosis that disappears without leaving any radiological residuals, while subclinical histoplasmosis often causes characteristic residuals, such as pulmonary granulomas and calcified mediastinal lymphomas. Many years after subclinical paracoccidiomycosis, the disease can be reactivated. In a series of patients who had immigrated to Spain, there was a mean reported latency of $14 \mathrm{yrs}$, ranging from 4 months to 60 yrs [3].

As demonstrated in the current patient, cervical and submandibular lymphadenopathy, which may be complicated by cutaneous or mucocutaneous fistulae, frequently accompany pulmonary disease. Pharyngeal ulcers and laryngitis causing throat pain and hoarseness, similar to that experienced by the present patient, are other common manifestations of the disease. In severe cases, the granulomatous laryngitis may even be mistaken for cancer [4].

Radiological manifestation of pulmonary disease is variable and ranges from alveolar to reticulo-nodular interstitial infiltrates. Frequently, perihilar butterfly-shaped opacities, cavitary lesions and traction bronchiectasies may be noted [5]. Since radiographic presentation is highly variable, paracoccidiomycosis may mimic TB, sarcoidosis, Wegener's granulomatosis or carcinoma and other systemic mycoses. Biopsies are often diagnostic because Grocott's hexamine-silver stains paracoccidioides, which can then easily be identified within epitheloid granulomas. Fungal cultures finally confirm the diagnosis. Serological tests are available, but they are time consuming, expensive and the antibody production can be suppressed in immunocompromised patients where the detection of the specific antigen may be more helpful. Serial antibody detection can be used to document the success of the antifungal therapy.

Left untreated, paracoccidiomycosis has a high mortality rate. In 1940, sulphonamides were used for the first time as a therapy that was successful in $\sim 60 \%$ of patients [2]. This was a 3 -yr therapy, which often resulted in a relapse when the treatment was terminated precociously. Sensitivity of paracoccidioides to sulphonamides may also explain the transitory recovery of the patient during her 2-week treatment with sulphamethoxazole in Brazil. Therapy with azole-derived antifungals increased the rate of success from 60 to $90 \%$. The current recommendation is a therapy with $100 \mathrm{mg}$ itraconazole b.i.d. over 6 months. With this regimen, a success rate of $93 \%$ has been reported [2]. An alternative treatment consists of ketoconazole $400 \mathrm{mg} \cdot \mathrm{day}^{-1}$ over 6-18 months. Even after successful elimination of the fungus, affected organs may remain permanently damaged by fibrosis and scarring. Laryngo-tracheal stenoses and scarring of the lung are wellknown, long-term sequelae.

Physicians in nonendemic areas should be aware of paracoccidiomycosis, since more cases are expected with increasing migration and international travel. Symptoms such as hoarseness, pharyngeal pain with mucosal ulcerations, cervical lymphadenopathy and chronic cough in patients with 
a history of travelling in South America should raise suspicion for this disease that may be reactivated after years of latent infection.

\section{REFERENCES}

1 Kayser FH, Bienz KA, Eckert J, Zinkernagel RM. Primäre Mykosen [Primary Mycosis]. In: Medizinische Mikrobiologie. 9. Auflage [Medical Microbiology. 9th Edn]. Stuttgart, Georg Thieme Verlag, 2001; pp. 372-376.

2 Taborda AB, Arechavala AI. Paracoccidioidomycosis. In: Sarosi GA, Davies SF, eds. Fungal Diseases of the Lung. 3rd Edn. Philadelphia, Lippincott Williams \& Wilkins, 2000; pp. 79-89.

3 Ajello L, Polonelli L. Imported paracoccidioidomycosis: a public health problem in non-endemic areas. Eur J Epidemiol 1985; 1: 160-165.

4 Sant'Anna GD, Mauri M, Arrarte JL, Camargo H Jr. Laryngeal manifestations of paracoccidioidomycosis (South American blastomycosis). Arch Otolaryngol Head Neck Surg 1999; 125: 1375-1378.

5 Bethlem EP, Capone D, Maranhao B, Carvalho CRR, Wanke B. Paracoccidioidomycosis. Curr Opin Pulm Med 1999; 5: 319-325. 